\title{
Interactive Effects of Snps Located Within CD28/B7Pathway and Environment on Susceptibility to Recurrent Spontaneous Abortion
}

\author{
Guiling Wang ${ }^{a}$ Jing Sun \\ ${ }^{a}$ Reproductive Medical Center, Affiliated Hospital of Weifang Medical University, Weifang City, \\ Shandong Province, ${ }^{b}$ Department of Obstetrics, Weifang People's Hospital, Weifang City, Shandong \\ Province, China
}

\section{Key Words}

Recurrent Spontaneous Abortion - CD28/B7 pathway - Environment Single Nucleotide Polymorphism

\begin{abstract}
Aims: This study was aimed to explore the interaction between environment and CD28/B7 pathway to provide the potential epidemiology for prevention and treatment of recurrent spontaneous abortion (RSA). Methods: The retrospective study included 630 RSA cases and 1320 healthy women during their middle and late prenatal care. Their living environment was investigated, and the influence of environmental factors on pregnancy abortion was analyzed. The genomic DNAs were extracted from the study subjects, and the polymorphisms of CD28 and B7 were analyzed. Finally, the interaction of gene and environment on RSA was analyzed with the logistic regression analyses. Results: The multi-variate regression analysis indicated that vitamin supplement, intake of fresh fruits or vegetables, night shift, staying up late, history miscarriage, as well as history induced abortion were, independently, risk factors for RSA (all $P<0.05)$. Moreover, rs3116496 (T>C), rs3181098 (G>A) and rs3181100 (G>C) of CD28, rs1915087 (C>T) of B7-2, as well as rs6804441 (A>G) and rs41271391 (G>T) of B7-1 were correlated with modified RSA risk (all $P<0.05$ ). The haplotypes TGT and TAG could also regulate the risk of RSA (both $P<0.05$ ). The synthetic influences of the aforementioned SNPs and environmental factors could also significantly affect the susceptibility to RSA (all $P<0.05$ ). Conclusion: The interaction of environment and SNPS of CD28/B7 pathway on RSA risk was distinct from CD28/B7 pathway or environment alone.
\end{abstract}




\section{Cellular Physiology Cell Physiol Biochem 2017;43:2185-2199

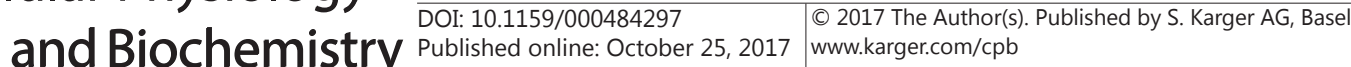

Wang/Sun: Role of CD28/B7 Pathway SNPs and Environment in RAS

\section{Introduction}

Spontaneous abortion was manifested as the termination of pregnancy before the $28^{\text {th }}$ week of pregnancy, while recurrent spontaneous abortion (RSA) was nominated when at least 2 times of terminated pregnancies were present before the $28^{\text {th }}$ week of pregnancy [1]. Of note, up to $5 \%$ of the infertile couples were induced by RAS, and RAS was found to account for $15 \sim 20 \%$ of all abortions in China [2]. Besides, the pregnancy abortion that did not accomplish any births before was called primary spontaneous abortion, whereas the secondary abortion was indicated as the abortion that occurred after at least a successful pregnancy. In line with the epidemiological investigations, the odds of pregnancy loss increased with the rising number of abortions, for instance, the probability of abortion could achieve $24 \%$ after 2 miscarriages, and it even stood high at $40 \%$ after 4 miscarriages [3].

Among the parameters that contributed to RSA, the abnormal functioning of the immune system was suspected to account for certain self-related and unexplained infertilities [4]. Specifically, during the breeding process, embryo, as an antigen, would stimulate the parent body to generate antibodies, when the parent body would form immunologic tolerance to be in a status of immunologic balance. However, once this equilibrium was broken, the spontaneous abortion would take place. It has been previously summarized that self-related immune factors mainly consisted of antiphospholipid antibody (APA), anti-sperm antibody (ASA) and so on, while isoimmunization was associated with humoral NK cells, T cells and so on. Activation of $\mathrm{T}$ cells necessitated not only the interaction of antigen-specific $\mathrm{T}$ cell receptors (TCRs) and MHC composite on the antigen presented cells (APCs), but also the costimulatory signal. The signals were mediated by the interplay of the co-stimulatory receptor with according ligands on the T cells, which were similar in their structures and belonged to the immunoglobulin superfamily.

CD28 was discovered by Hansen in 1980, and it was constitutively expressed on the surface of CD4+ T cells, CD8+ T cells, CD3+ peripheral blood T cells, plasmocytes and NK cells [5]. B7 molecules were mainly expressed on the surface of APCs, and they would provide the co-stimulatory signal when T cells were activated, thereby assisting in T cells' multiplication, secretion of cytokines, and production of cytotoxic effects [6]. For instance, when compared with wide-type mice, the proportion of regulatory T cells (Tregs) within the spleen of B7-1deficient and CD28-deficient rats, respectively, dropped to $10 \%$ and $25 \%$ [7]. Above all, the binding of CD28 and B7 could facilitate activation and clonally expansion of T cells, and highly expressed CTLA-4 within activated CD8 + T cells would maintain a balanced immune response through inhibiting over-proliferation of T cells [8]. Considering the vital role of CD28/B7 pathway in modifying immune responses and the crucial effects of immune dysfunctions on development of RSA, it was hypothesized that single nucleotide polymorphisms (SNPs) located within CD28 and B7 might contribute to differed consequences of RSA.

In addition, surroundings also seemed to contribute much to RSA. For example, the male animals that were exposed to phthalic acid esters (PAEs) in the early development of embryos were prone to develop such deformations as hypospadias and cryptorchism within their urinary systems [9]. Furthermore, an epidemiological survey based on female workers in the plastic industry found that their incidence of abnormal menstruation, menstrual cycle and menstrual blood volume were significantly higher than other normal industries, which were all hazard factors for menstruation [10].

To sum up, RSA appeared as the combined results of gene and environment, therefore, the current study was aimed to investigate the effects of CD28/B7 and CD28/B7-environment on susceptibility to RSA.

\section{Materials and Methods}

\section{Subjects}

This retrospective study included 630 RSA cases of Han ethnicities recruited from Affiliated Hospital of Weifang Medical University and Weifang People's Hospital from October 2013 to October 2016. They 
were diagnosed as spontaneous abortion after detection of serum human chorionic gonadotropin (HCG) via B-ultrasound measurement and/or electro-chemi-luminescence (ECL) within 13 gestational weeks. The criteria for ultrasonic diagnosis and detection of serum HCG were in accordance with the Ultrasound Diagnosis in Obstetrics and Gynecology published by People's Medical Publishing House. Besides, the Han pregnant women who received perinatal health care in the hospital were incorporated into the control group. Their gestation lasted longer than 13 weeks, and we failed to find any abnormality of fetal growth and development among the pregnant women. All the subjects have signed informed consents. This study was approved by Affiliated Hospital of Weifang Medical University and Weifang People's Hospital and the ethics committee of Affiliated Hospital of Weifang Medical University and Weifang People's Hospital.

\section{Investigation for pregnant women}

General conditions included name, birth, body mass index (BMI), career, family income and educational level. The fertility indicators included onset of menstruation, number of conception, frequency of abortion, menstrual condition, eugenics inspection and so on. The indicators relevant to living habit included usage of tableware, intake of milk, fruit, vegetable, puffed food, fried food and barbecue food, drinking, smoking, usage of television, telephone, microwave oven, electromagnetic oven and electric blanket, as well as the frequency of staying up late. The parameters of living condition included existences of decoration, passive smoking, factory pollution, noise, high-tension cable, signal beacon and traffic station. Additional conditions were also investigated, including medication before and during pregnancy, diseases, sexual life, emotion, working stress, physical training and so on.

\section{Definition of certain variables}

The subjects were confirmed with smoking history when they smoked one cigarette per day for 1 year. Night shift symbolized the work that sustained from $22 \mathrm{pm}$ to $6 \mathrm{am}$. The ones that did not rest until after 1 am were believed as staying up late and frequent staying up late indicates it happens at least 3 times a week. In addition, physical exercise should be continued for $>30 \mathrm{~min}$ each time, and the post-exercise heart rate should increase by $>30 \%$. Passive smoking represented that the subjects unconsciously inhaled the smoke for $\geq 15$ min each time.

\section{Clinical examination for the pregnant women}

The subjects all received blood examination, and they did not have abnormalities in chromosomes or thyroid function. Ultra-sound examination was performed to confirm that they did not have malformations in the genital tracts.

\section{Genotyping}

The vacuum tubes with ethylene diamine tetraacetic acid (EDTA) anti-coagulant were applied to extract $5 \mathrm{ml}$ peripheral blood from each subject, and then the blood samples were preserved in the -80 ${ }^{\circ} \mathrm{C}$ refrigerator. Genomic DNA was extracted with assistance of the DNA extraction kit (ComWin Biotech Co.,Ltd., Beijing, China). In line with the genetic sequence provided by Gene Bank database, the primers were designed based on Premier 5 software (Table 1), and were synthesized by Dalian Takara Biological Engineering Co., Ltd..

Table 1. Primers of the investigated single nucleotide polymorphisms (SNPs)

\begin{tabular}{|c|c|c|c|c|}
\hline \multirow{2}{*}{ Gene } & \multirow{2}{*}{ SNP } & \multicolumn{2}{|c|}{ Primers } & \multirow{2}{*}{ Restriction enzyme } \\
\hline & & Sense & Anti-sense & \\
\hline \multirow[t]{6}{*}{ CD28 } & rs3116496 & 5'-ATAGCTTGCTAGTAACAGT-3' & 5'-AAAATAAACCACATAGGC-3' & AciI \\
\hline & rs3769684 & 5'-AATAGGTGTAAACGGCAAGA-3' & 5'-ACAGCCGACTTCAGGAAA-3' & CviAII \\
\hline & rs3181098 & 5'-AAATGCTCCAGAGGGCTAC-3' & 5'-TTGGGAAACATCTGCATAAA-3' & AciI \\
\hline & rs3181100 & 5'-TGCTGTAAGTAGATTGGC-3' & 5'-GAAACTGAGGTTTGGAGA-3' & DdeI \\
\hline & rs4673259 & 5'-ATGGGAAGACTTTCAAGA-3' & 5'-TCTGTGGTCATTTCATCT-3' & Нру188III \\
\hline & rs10932017 & 5'-GAAGCAGCGGAGAATCCA-3' & 5'-СTTCTTACCCAGGCCATT-3' & DdeI \\
\hline \multirow[t]{4}{*}{ B7-2 } & rs1129055 & 5'-ТTTTCTTCTATTTCTCCAGA-3' & 5'-ATGTATCACTTTTGTCGC-3' & CvikI-I \\
\hline & rs17281995 & 5'-TTACTTAGCCTACAGATGTCC-3' & 5'-TTTCСТCTGGTTGCCTTG-3' & MluCI \\
\hline & rs1915087 & 5'-AAACTGCCTTTTATCTGC-3' & 5'-ATACATAAGTCCCACCCA-3' & ApoI \\
\hline & rs9282641 & 5'-CCGTCAGTCCTGGCATTA-3' & 5'-CACAGAAGCAAGGTGGTA-3' & CviQI \\
\hline \multirow[t]{3}{*}{ B7-1 } & rs6804441 & 5'-TGTAATTAACTAATACAGGCTA-3' & 5'-AGTTTCTCCATTTTCCTC-3' & MseI \\
\hline & rs41271391 & 5'-GCGACATTTCСССТTCTA-3' & 5'-CGCTTCTTCAAACACCСT-3' & MspJI \\
\hline & rs16829984 & 5'-TGCTGCCGTCTTCTTCTA-3' & 5'-CAGCCACAGCCTCTACAA-3' & BfaI \\
\hline
\end{tabular}


Genotyping was analyzed based on polymerase chain reaction-restriction fragment length polymorphism (PCR-RFLP). The PCR amplification system $(20 \mu \mathrm{l})$ included forward primer $(2 \mu \mathrm{l})$, reverse primer $(2 \mu \mathrm{l})$, DNA template $(<1 \mu \mathrm{g}), 2 \times$ EsTaq Master Mix $(25 \mu \mathrm{l})$ and RNase-free water (up to $50 \mu \mathrm{l}$ ). The PCR reaction conditions were arranged as: 1) pre-degeneration at $94^{\circ} \mathrm{C}$ for $15 \mathrm{~min}$; 2) 45 cycles of degeneration at $95{ }^{\circ} \mathrm{C}$ for $30 \mathrm{~s}$, annealing at $56^{\circ} \mathrm{C}$ for $30 \mathrm{~s}$, and extension at $72{ }^{\circ} \mathrm{C}$ for $30 \mathrm{~s}$; and 3) eventual extension at $72^{\circ} \mathrm{C}$ for $5 \mathrm{~min}$. Then $10 \mu \mathrm{l}$ PCR products were added with $5 \mathrm{U}$ endonuclease, $2 \mu \mathrm{l} 10 \times$ enzyme buffer and $20 \mu \mathrm{lddH} 20$, forming the $20 \mu \mathrm{l}$ reaction system. Digestion was conducted within the water bath at the temperature of $37^{\circ} \mathrm{C}$ for $2 \sim 16 \mathrm{~h}$, and then the electrophoretic results were observed using gel imager (model: Gel Doc 2000; manufacturer: Bio-RAD corporation). The sequencing of PCR results was accomplished by Shanghai sangon biological engineering corporation.

\section{Statistical analyses}

All the statistical analyses were performed with assistance of the SPSS 17.0 software. The associations of genotypes with early abortion were analyzed with univariate logistic regression analysis. Moreover, the parameters of vitamin supplement, intake of fresh fruit or vegetables, alcohol consumption, frequency of smoking, frequency of night shift, frequency of physical exercise, as well as history of miscarriage were adjusted by way of multivariate logistic regression analysis. The correlation degrees were expressed by odds ratios (ORs) and their 95\% confidence intervals (CIs).

\section{Results}

Effect of certain living habits on the prevalence of RSA

As was shown in Table 2, vitamin supplement, intake of fresh fruits or vegetables, as well as regular physical exercise were all, respectively, correlated with reduced incidence of RSA (OR = 0.75, 95\% CI: 0.62-0.90, $P<0.05$; OR = 0.60, 95\% CI: 0.48-0.75, $P<0.05 ; 0 R=0.58$, 95\% CI: 0.48-0.71, $P<0.05)$. In contrast, frequent night shift, frequent staying up late, history of miscarriage and previously induced abortion all appeared to elevate the possibility of suffering from RSA (OR = 1.23, 95\% CI: 1.01-1.48, $P<0.05$; OR = 1.67, 95\% CI: 1.36-2.05, $P<0.05 ;$ OR $=2.94,95 \%$ CI: 2.42-3.58, $P<0.05 ;$ OR $=2.09,95 \% \mathrm{CI}: 1.72-2.53, P<0.05)$. Nonetheless, the results of multivariate analysis (Table 3 ) indicated that vitamin supplement,

Table 2. Effect of certain living habits on the prevalence of recurrent spontaneous abortion

\begin{tabular}{|c|c|c|c|c|c|c|}
\hline \multirow{2}{*}{ Living Habits } & \multicolumn{2}{|c|}{ Case } & \multicolumn{2}{|c|}{ Control } & \multirow{2}{*}{ OR } & \multirow{2}{*}{$95 \%$ CI } \\
\hline & Number & Percent & Number & Percent & & \\
\hline \multicolumn{7}{|c|}{ Vitamin supplement } \\
\hline Yes & 339 & $53.81 \%$ & 805 & $60.98 \%$ & & \\
\hline No & 291 & $46.19 \%$ & 515 & $39.02 \%$ & 0.75 & $0.62-0.90$ \\
\hline \multicolumn{7}{|c|}{ Fresh fruit or vegetables daily or most days } \\
\hline Yes & 453 & $71.90 \%$ & 1069 & $80.98 \%$ & & \\
\hline No & 177 & $28.10 \%$ & 251 & $19.02 \%$ & 0.60 & $0.48-0.75$ \\
\hline \multicolumn{7}{|c|}{ Alcohol consumption } \\
\hline Yes & 94 & $14.92 \%$ & 158 & $11.97 \%$ & & \\
\hline No & 536 & $85.08 \%$ & 1162 & $88.03 \%$ & 1.29 & $0.98-1.70$ \\
\hline \multicolumn{7}{|c|}{ Smoking before and in the first $12 \mathrm{wk}$ of pregnancy (No. of cigarettes) } \\
\hline No smoking & 609 & $96.67 \%$ & 1284 & $97.27 \%$ & & \\
\hline $1-10 / d$ & 16 & $2.54 \%$ & 28 & $2.12 \%$ & 1.21 & $0.65-2.24$ \\
\hline $11-20 / d$ & 4 & $0.63 \%$ & 7 & $0.53 \%$ & 1.22 & $0.35-4.13$ \\
\hline$>20 / d$ & 1 & $0.16 \%$ & 1 & $0.08 \%$ & 2.11 & $0.13-33.79$ \\
\hline \multicolumn{7}{|c|}{ Frequency of night shift } \\
\hline$\leq 1$ times/wk & 302 & $47.94 \%$ & 700 & $53.03 \%$ & & \\
\hline$\geq 2$ times/wk & 328 & $52.06 \%$ & 620 & $46.97 \%$ & 1.23 & $1.01-1.48$ \\
\hline \multicolumn{7}{|c|}{ Frequent staying up late } \\
\hline Yes & 233 & $36.98 \%$ & 343 & $25.98 \%$ & & \\
\hline No & 397 & $63.02 \%$ & 977 & $74.02 \%$ & 1.67 & $1.36-2.05$ \\
\hline \multicolumn{7}{|c|}{ Regular physical exercise } \\
\hline Yes & 323 & $51.27 \%$ & 849 & $64.32 \%$ & & \\
\hline No & 307 & $48.73 \%$ & 471 & $35.68 \%$ & 0.58 & $0.48-0.71$ \\
\hline \multicolumn{7}{|c|}{ History of miscarriage } \\
\hline Yes & 344 & $54.60 \%$ & 383 & $29.02 \%$ & & \\
\hline No & 286 & $45.40 \%$ & 937 & $70.98 \%$ & 2.94 & $2.42-3.58$ \\
\hline \multicolumn{7}{|c|}{ Previous induced abortion } \\
\hline Yes & 309 & $49.05 \%$ & 417 & $31.59 \%$ & & \\
\hline No & 321 & $50.95 \%$ & 903 & $68.41 \%$ & 2.09 & $1.72-2.53$ \\
\hline
\end{tabular}


Table 3. Results of multivariate analysis between living habits and the prevalence of recurrent spontaneous abortion

\begin{tabular}{lccc}
\hline Living Habits & OR & $95 \%$ CI & P value \\
\hline Vitamin supplement & 0.13 & $0.02-0.98$ & 0.05 \\
Fresh fruit or vegetables & 0.39 & $0.20-0.78$ & 0.01 \\
Alcohol consumption & 0.7 & $0.47-1.05$ & 0.09 \\
Smoking & 0.44 & $0.19-1.04$ & 0.06 \\
Frequency of night shift & 1.78 & $1.04-3.04$ & 0.04 \\
Frequent staying up late & 1.63 & $1.05-2.52$ & 0.03 \\
Regular physical exercise & 0.25 & $0.03-1.92$ & 0.18 \\
History of miscarriage & 166.67 & $23.26-1000$ & $<0.01$ \\
History of induced abortion & 36.06 & $4.66-279.02$ & 0.01 \\
Constant & 7.14 & & $<0.01$ \\
\hline
\end{tabular}

Table 4. Association of CD28/B7 pathway-related single nucleotide polymorphisms with susceptibility to recurrent spontaneous abortion

\begin{tabular}{|c|c|c|c|c|c|c|c|c|}
\hline Gene & SNP & Allele Change & Genotype & Case Group & Control Group & OR & $95 \% \mathrm{CI}$ & $\mathrm{P}$ value \\
\hline \multirow[t]{24}{*}{ CD28 } & rs3116496 & $\mathrm{T}>\mathrm{C}$ & $\mathrm{T}$ & 969 & 2244 & 1.00 & & \\
\hline & & & $\mathrm{C}$ & 291 & 396 & 1.70 & $1.44-2.02$ & $<0.01$ \\
\hline & & & Dominant model & & & 1.78 & $1.46-2.18$ & $<0.01$ \\
\hline & & & Recessive model & & & 2.53 & $1.54-4.16$ & $<0.01$ \\
\hline & rs3769684 & $\mathrm{T}>\mathrm{C}$ & $\mathrm{T}$ & 1058 & 2164 & 1.00 & & \\
\hline & & & $\mathrm{C}$ & 202 & 476 & 0.87 & $0.72-1.04$ & 0.12 \\
\hline & & & Dominant model & & & 0.85 & $0.69-1.05$ & 0.13 \\
\hline & & & Recessive model & & & 0.82 & $0.47-1.46$ & 0.50 \\
\hline & rs3181098 & $\mathrm{G}>\mathrm{A}$ & G & 731 & 1636 & 1.00 & & \\
\hline & & & $\mathrm{A}$ & 529 & 1004 & 1.18 & $1.03-1.35$ & 0.02 \\
\hline & & & Dominant model & & & 1.23 & $1.01-1.50$ & 0.04 \\
\hline & & & Recessive model & & & 1.26 & $0.98-1.63$ & 0.07 \\
\hline & rs3181100 & $\mathrm{G}>\mathrm{C}$ & G & 314 & 819 & 1.00 & & \\
\hline & & & $\mathrm{C}$ & 946 & 1821 & 1.35 & $1.16-1.58$ & $<0.01$ \\
\hline & & & Dominant model & & & 1.61 & $1.11-2.34$ & 0.01 \\
\hline & & & Recessive model & & & 1.42 & $1.18-1.72$ & $<0.01$ \\
\hline & rs4673259 & $\mathrm{T}>\mathrm{C}$ & $\mathrm{T}$ & 1034 & 2217 & 1.00 & & \\
\hline & & & $\mathrm{C}$ & 226 & 423 & 1.15 & $0.96-1.37$ & 0.13 \\
\hline & & & Dominant model & & & 1.16 & $0.95-1.43$ & 0.15 \\
\hline & & & Recessive model & & & 1.24 & $0.71-2.17$ & 0.45 \\
\hline & rs10932017 & $\mathrm{C}>\mathrm{T}$ & $\mathrm{C}$ & 654 & 1426 & 1.00 & & \\
\hline & & & $\mathrm{T}$ & 606 & 1214 & 1.09 & $0.95-1.25$ & 0.22 \\
\hline & & & Dominant model & & & 1.11 & $0.90-1.38$ & 0.32 \\
\hline & & & Recessive model & & & 1.13 & $0.90-1.41$ & 0.31 \\
\hline \multirow[t]{16}{*}{ B7-2 } & rs1129055 & $\mathrm{G}>\mathrm{A}$ & G & 1071 & 2297 & 1.00 & & \\
\hline & & & $\mathrm{A}$ & 189 & 343 & 1.18 & $0.98-1.43$ & 0.09 \\
\hline & & & Dominant model & & & 1.20 & $0.97-1.48$ & 0.10 \\
\hline & & & Recessive model & & & 1.34 & $0.68-2.64$ & 0.39 \\
\hline & rs17281995 & $\mathrm{G}>\mathrm{C}$ & G & 367 & 819 & 1.00 & & \\
\hline & & & $\mathrm{C}$ & 893 & 1821 & 1.09 & $0.94-1.27$ & 0.23 \\
\hline & & & Dominant model & & & 1.14 & $0.81-1.59$ & 0.46 \\
\hline & & & Recessive model & & & 1.12 & $0.92-1.35$ & 0.26 \\
\hline & rs1915087 & $\mathrm{C}>\mathrm{T}$ & $\mathrm{C}$ & 404 & 738 & 1.00 & & \\
\hline & & & $\mathrm{T}$ & 856 & 1902 & 0.82 & $0.71-0.95$ & 0.01 \\
\hline & & & Dominant model & & & 0.74 & $0.53-1.02$ & 0.06 \\
\hline & & & Recessive model & & & 0.80 & $0.66-0.96$ & 0.02 \\
\hline & rs9282641 & $\mathrm{G}>\mathrm{A}$ & G & 781 & 1557 & 1.00 & & \\
\hline & & & $\mathrm{A}$ & 479 & 1083 & 0.88 & $0.77-1.01$ & 0.07 \\
\hline & & & Dominant model & & & 0.85 & $0.70-1.04$ & 0.12 \\
\hline & & & Recessive model & & & 0.84 & $0.64-1.09$ & 0.18 \\
\hline \multirow[t]{12}{*}{ B7-1 } & rs6804441 & $A>G$ & $\mathrm{~A}$ & 328 & 450 & 1.00 & & \\
\hline & & & G & 932 & 2190 & 0.58 & $0.50-0.69$ & $<0.01$ \\
\hline & & & Dominant model & & & 0.42 & $0.27-0.65$ & $<0.01$ \\
\hline & & & Recessive model & & & 0.55 & $0.45-0.67$ & $<0.01$ \\
\hline & rs41271391 & $\mathrm{G}>\mathrm{T}$ & G & 541 & 950 & 1.00 & & \\
\hline & & & $\mathrm{T}$ & 719 & 1690 & 0.75 & $0.65-0.86$ & $<0.01$ \\
\hline & & & Dominant model & & & 0.66 & $0.51-0.85$ & $<0.01$ \\
\hline & & & Recessive model & & & 0.69 & $0.57-0.85$ & $<0.01$ \\
\hline & rs16829984 & $\mathrm{G}>\mathrm{C}$ & G & 1133 & 2348 & 1.00 & & \\
\hline & & & $\mathrm{C}$ & 127 & 292 & 0.90 & $0.72-1.12$ & 0.35 \\
\hline & & & Dominant model & & & 0.89 & $0.70-1.14$ & 0.36 \\
\hline & & & Recessive model & & & 0.86 & $0.36-2.09$ & 0.74 \\
\hline
\end{tabular}

intake of fresh fruits or vegetables, night shift, staying up late, history miscarriage, as well as history induced abortion could independently regulate the incidence of RSA.

Association of CD28/B7 pathway-related SNPs and haplotypes with susceptibility to RSA

Interestingly, rs3116496 ( $\mathrm{T}>\mathrm{C}), \mathrm{rs} 3181098(\mathrm{G}>\mathrm{A})$ and $\mathrm{rs} 3181100(\mathrm{G}>\mathrm{C})$ of CD28 were, respectively, found to be correlated with incremental susceptibility to RSA under the allelic model $(\mathrm{OR}=1.70,95 \% \mathrm{CI}: 1.44-2.02, P<0.05 ; \mathrm{OR}=1.18,95 \% \mathrm{CI}: 1.03-1.35, P<0.05$; OR = 
1.35, 95\% CI: 1.16-1.58, $P<0.05$ ), while rs1915087 (C>T), rs6804441 (A>G) and rs41271391 $(\mathrm{G}>\mathrm{T})$ of $\mathrm{B} 7$ were suspected as the factors associated with reduced risk of RSA (OR $=0.82$, 95\% CI: 0.71-0.95, $P<0.05$; OR $=0.58,95 \%$ CI: 0.50-0.69, $P<0.05$; OR $=0.75,95 \%$ CI: $0.65-$ $0.86, P<0.05$ )(Table 4). Furthermore, for rs3116496, the remarkably enhancive RSA risk could also be found among the individuals with genotypes CC/TC, when compared with ones carrying homozygote TT $(\mathrm{OR}=1.78,95 \% \mathrm{CI}: 1.46-2.18, P<0.05)$. Additionally, subjects carrying genotypes AA/GA of rs3181098 or genotypes CC/GC of rs3181100 were more prone to suffer from RSA than ones carrying homozygote GG (OR $=1.23,95 \%$ CI: $1.01-1.50$, $P<0.05 ;$ OR $=1.61,95 \% \mathrm{CI}: 1.11-2.34, P<0.05$ ). Slightly different from the result of allelic model, it was shown in the dominant model that merely genotypes GG/GA of rs6804441 and genotypes TT/GT of rs41271391 would significantly lessen the RSA risk in comparison to homozygotes GG and CC (OR $=0.42,95 \%$ CI: 0.27-0.65, $P<0.05$; OR $=0.66,95 \%$ CI: 0.51-0.85, $P<0.05$ ) (Fig. 1).

However, there showed no significant distinctions between RSA cases and healthy controls $(P>0.05)$, regarding the genotyping and allelic distributions of rs3769684 $(\mathrm{T}>\mathrm{C})$, rs4673259 (T>C), rs10932017 (C>T), rs1129055 (G>A), rs17281995 (G>C), rs9282641 $(\mathrm{G}>\mathrm{A})$ and rs16829984 $(\mathrm{G}>\mathrm{C})$. Moreover, the haplotype TGT of B7 were suggested as the protective parameter for RSA (OR $=0.65,95 \% \mathrm{CI}: 0.53-0.80, P<0.05)$, whereas the haplotype TAG seemed as a risky element for facilitating development of RSA (OR = 1.79, 95\% CI: 1.212.66, $P<0.05$ )(Table 5).

Fig. 1. The electrophoretic results for genotyping of eligible SNPs .

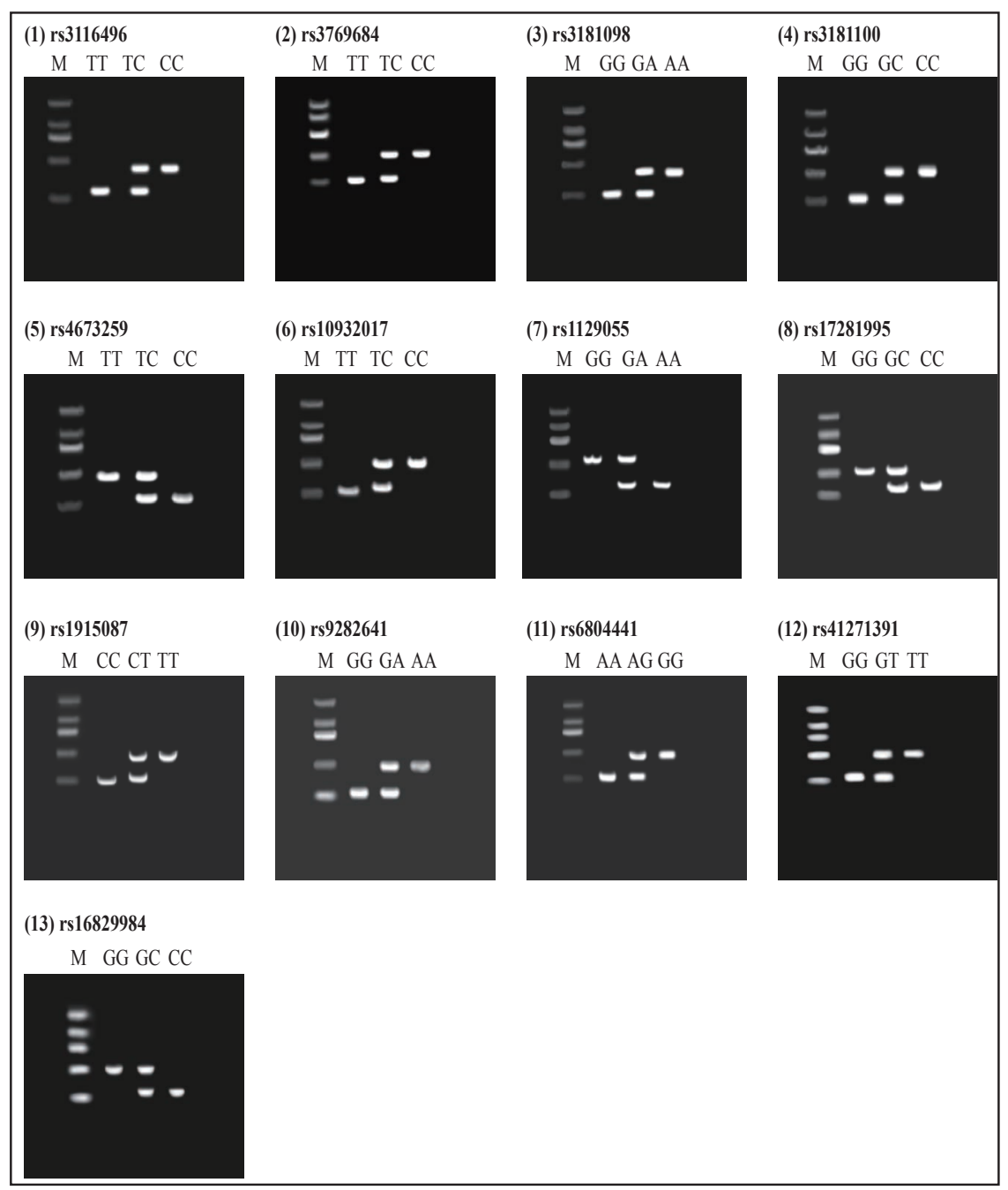


Table 5. Association of CD28/B7 pathway-related haplotypes with susceptibility to recurrent spontaneous abortion

\begin{tabular}{lcccccc}
\hline Gene & Haplotypes & Case & Control & OR & $95 \%$ CI & P value \\
\hline CD28 & TGC & 211 & 480 & 0.88 & $0.72-1.08$ & 0.22 \\
B7 & CGT & 85 & 196 & 0.89 & $0.68-1.18$ & 0.43 \\
& CGG & 64 & 110 & 1.24 & $0.90-1.72$ & 0.19 \\
& CAT & 30 & 40 & 1.60 & $0.99-2.59$ & 0.05 \\
& TGT & 181 & 505 & 0.65 & $0.53-0.80$ & $<0.01$ \\
& TGG & 136 & 284 & 1.00 & $0.80-1.26$ & 0.97 \\
& TAT & 63 & 103 & 1.31 & $0.94-1.82$ & 0.10 \\
& TAG & 48 & 58 & 1.79 & $1.21-2.66$ & $<0.01$ \\
\hline
\end{tabular}

Table 6. Interactive effects of CD28/B7 pathway-related SNPs and vitamin supplement on susceptibility to recurrent spontaneous abortion

\begin{tabular}{|c|c|c|c|c|c|c|c|c|}
\hline 6. Int & SNP & Genotype & Vitamin supplement & Case & Control & OR & $95 \% \mathrm{CI}$ & $P$ value \\
\hline of $\mathrm{CD} 28 / \mathrm{B} 7$ path- & rs3116496 & $\mathrm{CC}$ & Yes & 8 & 6 & 2.70 & $0.92-7.91$ & 0.06 \\
\hline & & $\mathrm{CC}$ & No & 27 & 24 & 2.28 & $1.27-4.07$ & $<0.01$ \\
\hline lated SNPS & & $\mathrm{TC}$ & Yes & 118 & 171 & 1.40 & $1.03-1.89$ & 0.03 \\
\hline supplement & & $\mathrm{TC}$ & No & 103 & 165 & 1.26 & $0.93-1.72$ & 0.14 \\
\hline & & TT & Yes & 213 & 628 & 0.69 & $0.54-0.88$ & $<0.01$ \\
\hline ecurrent & & TT & No & 161 & 326 & 1.00 & & \\
\hline ineous abortion & rs3181098 & $\mathrm{AA}$ & Yes & 21 & 50 & 0.84 & $0.48-1.48$ & 0.56 \\
\hline & & AA & No & 90 & 141 & 1.28 & $0.90-1.84$ & 0.17 \\
\hline & & GA & Yes & 204 & 445 & 0.92 & $0.69-1.24$ & 0.59 \\
\hline & & GA & No & 103 & 177 & 1.17 & $0.83-1.65$ & 0.37 \\
\hline & & GG & Yes & 114 & 310 & 0.74 & $0.53-1.02$ & 0.07 \\
\hline & & GG & No & 98 & 197 & 1.00 & & \\
\hline interactive & rs3181100 & $\mathrm{CC}$ & Yes & 169 & 264 & 1.28 & $0.62-2.63$ & 0.50 \\
\hline ffects of CD28/ & & CC & No & 186 & 364 & 1.02 & $0.50-2.09$ & 0.95 \\
\hline ejJecis oj CDLo/ & & GC & Yes & 143 & 438 & 0.65 & $0.32-1.34$ & 0.24 \\
\hline B7 pathway- & & GC & No & 93 & 127 & 1.46 & $0.70-3.08$ & 0.31 \\
\hline related SNPS and & & GG & Yes & 27 & 103 & 0.52 & $0.23-1.18$ & 0.12 \\
\hline & & GG & No & 12 & 24 & 1.00 & & \\
\hline ving habits on & rs1915087 & TT & Yes & 118 & 441 & 0.39 & $0.22-0.69$ & $<0.01$ \\
\hline susceptibility to & & TT & No & 173 & 244 & 1.03 & $0.58-1.84$ & 0.92 \\
\hline & & CT & Yes & 178 & 293 & 0.88 & $0.50-1.57$ & 0.67 \\
\hline & & СT & No & 96 & 239 & 0.58 & $0.32-1.06$ & 0.07 \\
\hline results & & CC & Yes & 43 & 71 & 0.88 & $0.45-1.71$ & 0.71 \\
\hline plained 1 & & $\mathrm{CC}$ & No & 22 & 32 & 1.00 & & \\
\hline spamea & rs6804441 & GG & Yes & 152 & 513 & 0.27 & $0.12-0.63$ & $<0.01$ \\
\hline supplement & & GG & No & 193 & 396 & 0.45 & $0.19-1.03$ & 0.05 \\
\hline could effectively & & $\mathrm{AG}$ & Yes & 156 & 264 & 0.54 & $0.23-1.26$ & 0.15 \\
\hline & & AG & No & 86 & 108 & 0.73 & $0.31-1.73$ & 0.47 \\
\hline down & & AA & Yes & 31 & 28 & 1.01 & $0.39-2.66$ & 0.98 \\
\hline nce of & & AA & No & 12 & 11 & 1.00 & & \\
\hline U1 & rs41271391 & TT & Yes & 99 & 197 & 0.38 & $0.23-0.61$ & $<0.01$ \\
\hline carriers & & TT & No & 106 & 344 & 0.23 & $0.14-0.37$ & $<0.01$ \\
\hline 16496 TT (OR & & GT & Yes & 176 & 476 & 0.28 & $0.18-0.43$ & $<0.01$ \\
\hline 10 & & GT & No & 133 & 132 & 0.76 & $0.47-1.22$ & 0.25 \\
\hline & & GG & Yes & 64 & 132 & 0.36 & $0.22-0.61$ & $<0.01$ \\
\hline$P<0.05)$, an & & GG & No & 52 & 39 & 1.00 & & \\
\hline
\end{tabular}
homozygote CC was implied as the hazard factor for RSA among the ones without vitamin supplement $(\mathrm{OR}=2.28$, $95 \%$ CI: $1.27-4.07, P<0.05)$. Besides, the risky role of rs3116496 TC seemed to overweigh the protective function of vitamin supplement in contributing to RSA (OR $=1.40,95 \% \mathrm{CI}$ : $1.03-1.89, P<0.05)$. Moreover, the combined influence of rs1915087 TT, rs6804441 GG or rs41271391 TT and vitamin supplement were associated with largely reduced RSA risk (OR $=0.39,95 \%$ CI: 0.22-0.69, $P<0.05 ;$ OR $=0.27,95 \%$ CI: 0.12-0.63, $P<0.05 ;$ OR $=0.38,95 \%$ CI: 0.23-0.61, $P<0.05$ ).

As for the subjects who frequently ingested fresh vegetables and fruits (Table 7), carriers of rs3116496 TT were less probable to develop RSA than ones without intake of fresh vegetables or fruits (OR $=0.38,95 \% \mathrm{CI}: 0.29-0.50, P<0.05$ ). Besides, the heterozygote TC of rs3116496 was also associated with decreased susceptibility to RSA, yet its homozygote CC was associated with elevated RSA risk (OR $=4.42$, 95\% CI: 1.60-12.22, $P<0.05)$. Similarly, rs3181098 AA and rs3181100 CC were tightly linked with the raised prevalence of RSA, considering the crowds who took vegetables and fruits marginally $(\mathrm{OR}=2.90,95 \% \mathrm{CI}$ : 1.63 5.15, $P<0.05$; OR $=2.31,95 \% \mathrm{CI}: 1.06-5.03, P<0.05$ ).

In addition, carriers of rs3116496 CC were more readily to suffer from RSA than carriers of TT, whether they experienced night shift or not $(\mathrm{OR}=2.21,95 \% \mathrm{CI}: 1.06-4.62, P<$ 
Table 7. Interactive effects of CD28/B7 pathway-related SNPs and fresh fruit or vegetables on susceptibility to recurrent spontaneous abortion

\begin{tabular}{|c|c|c|c|c|c|c|c|}
\hline SNP & Genotype & Fresh fruit or vegetables & Case & Control & OR & $95 \% \mathrm{CI}$ & P value \\
\hline \multirow{6}{*}{ rs3116496 } & $\mathrm{CC}$ & Yes & 17 & 25 & 0.83 & $0.43-1.61$ & 0.59 \\
\hline & $\mathrm{CC}$ & No & 18 & 5 & 4.42 & $1.60-12.22$ & $<0.01$ \\
\hline & $\mathrm{TC}$ & Yes & 190 & 247 & 0.94 & $0.70-1.27$ & 0.70 \\
\hline & $\mathrm{TC}$ & No & 31 & 89 & 0.43 & $0.27-0.68$ & $<0.01$ \\
\hline & TT & Yes & 246 & 797 & 0.38 & $0.29-0.50$ & $<0.01$ \\
\hline & TT & No & 128 & 157 & 1.00 & & \\
\hline \multirow[t]{6}{*}{ rs3181098 } & AA & Yes & 68 & 155 & 1.06 & $0.67-1.69$ & 0.79 \\
\hline & $\mathrm{AA}$ & No & 43 & 36 & 2.90 & $1.63-5.15$ & $<0.01$ \\
\hline & GA & Yes & 213 & 504 & 1.02 & $0.69-1.53$ & 0.90 \\
\hline & GA & No & 94 & 118 & 1.93 & $1.22-3.05$ & $<0.01$ \\
\hline & GG & Yes & 172 & 410 & 1.02 & $0.68-1.53$ & 0.93 \\
\hline & GG & No & 40 & 97 & 1.00 & & \\
\hline \multirow[t]{6}{*}{ rs3181100 } & $\mathrm{CC}$ & Yes & 245 & 509 & 1.20 & $0.57-2.55$ & 0.63 \\
\hline & $\mathrm{CC}$ & No & 110 & 119 & 2.31 & $1.06-5.03$ & 0.03 \\
\hline & GC & Yes & 179 & 458 & 0.98 & $0.46-2.08$ & 0.95 \\
\hline & $\mathrm{GC}$ & No & 57 & 107 & 1.33 & $0.60-2.97$ & 0.48 \\
\hline & GG & Yes & 29 & 102 & 0.71 & $0.31-1.65$ & 0.43 \\
\hline & GG & No & 10 & 25 & 1.00 & & \\
\hline \multirow[t]{6}{*}{ rs1915087 } & TT & Yes & 193 & 535 & 0.54 & $0.27-1.09$ & 0.08 \\
\hline & TT & No & 98 & 150 & 0.98 & $0.48-2.02$ & 0.96 \\
\hline & $\mathrm{CT}$ & Yes & 209 & 452 & 0.69 & $0.35-1.39$ & 0.30 \\
\hline & $\mathrm{CT}$ & No & 65 & 80 & 1.22 & $0.57-2.58$ & 0.61 \\
\hline & $\mathrm{CC}$ & Yes & 51 & 82 & 0.93 & $0.44-2.00$ & 0.86 \\
\hline & $\mathrm{CC}$ & No & 14 & 21 & 1.00 & & \\
\hline \multirow[t]{6}{*}{ rs6804441 } & GG & Yes & 271 & 737 & 0.59 & $0.19-1.81$ & 0.35 \\
\hline & GG & No & 74 & 172 & 0.69 & $0.22-2.17$ & 0.52 \\
\hline & $\mathrm{AG}$ & Yes & 144 & 301 & 0.77 & $0.25-2.38$ & 0.64 \\
\hline & $A G$ & No & 98 & 71 & 2.21 & $0.69-7.03$ & 0.17 \\
\hline & $\mathrm{AA}$ & Yes & 38 & 31 & 1.96 & $0.58-6.60$ & 0.27 \\
\hline & $\mathrm{AA}$ & No & 5 & 8 & 1.00 & & \\
\hline \multirow[t]{6}{*}{ rs41271391 } & TT & Yes & 137 & 439 & 0.57 & $0.31-1.06$ & 0.07 \\
\hline & TT & No & 68 & 102 & 1.22 & $0.62-2.37$ & 0.57 \\
\hline & GT & Yes & 217 & 490 & 0.81 & $0.44-1.49$ & 0.49 \\
\hline & GT & No & 90 & 118 & 1.39 & $0.72-2.67$ & 0.32 \\
\hline & $\mathrm{GG}$ & Yes & 99 & 140 & 1.29 & $0.68-2.46$ & 0.44 \\
\hline & GG & No & 17 & 31 & 1.00 & & \\
\hline
\end{tabular}

Table 8. Interactive effects of CD28/B7 pathway-related SNPs and night shift on susceptibility to recurrent spontaneous abortion

\begin{tabular}{|c|c|c|c|c|c|c|c|}
\hline SNP & Genotype & Night shift & Case & Control & OR & $95 \% \mathrm{CI}$ & P value \\
\hline \multirow[t]{6}{*}{ rs3116496 } & $\mathrm{CC}$ & Yes & 14 & 16 & 2.21 & $1.06-4.62$ & 0.03 \\
\hline & $\mathrm{CC}$ & No & 21 & 14 & 3.78 & $1.88-7.61$ & $<0.01$ \\
\hline & $\mathrm{TC}$ & Yes & 92 & 178 & 1.30 & $0.96-1.77$ & 0.09 \\
\hline & TC & No & 129 & 158 & 2.06 & $1.54-2.75$ & $<0.01$ \\
\hline & $\mathrm{TT}$ & Yes & 196 & 505 & 0.98 & $0.77-1.24$ & 0.86 \\
\hline & TT & No & 178 & 449 & 1.00 & & \\
\hline \multirow[t]{6}{*}{ rs3181098 } & AA & Yes & 54 & 101 & 1.15 & $0.77-1.71$ & 0.50 \\
\hline & $\mathrm{AA}$ & No & 57 & 90 & 1.36 & $0.91-2.03$ & 0.13 \\
\hline & GA & Yes & 147 & 330 & 0.96 & $0.71-1.29$ & 0.76 \\
\hline & GA & No & 160 & 292 & 1.17 & $0.87-1.58$ & 0.29 \\
\hline & GG & Yes & 101 & 269 & 0.81 & $0.58-1.11$ & 0.19 \\
\hline & GG & No & 111 & 238 & 1.00 & & \\
\hline \multirow[t]{6}{*}{ rs3181100 } & $\mathrm{CC}$ & Yes & 172 & 333 & 1.29 & $0.78-2.15$ & 0.32 \\
\hline & $\mathrm{CC}$ & No & 183 & 295 & 1.55 & $0.93-2.58$ & 0.09 \\
\hline & GC & Yes & 115 & 300 & 0.96 & $0.57-1.61$ & 0.87 \\
\hline & GC & No & 121 & 265 & 1.14 & $0.68-1.92$ & 0.62 \\
\hline & GG & Yes & 15 & 67 & 0.56 & $0.27-1.17$ & 0.12 \\
\hline & GG & No & 24 & 60 & 1.00 & & \\
\hline \multirow[t]{6}{*}{ rs1915087 } & TT & Yes & 138 & 363 & 0.61 & $0.37-1.00$ & 0.05 \\
\hline & $\mathrm{TT}$ & No & 153 & 322 & 0.76 & $0.46-1.25$ & 0.28 \\
\hline & $\mathrm{CT}$ & Yes & 129 & 282 & 0.73 & $0.44-1.21$ & 0.22 \\
\hline & CT & No & 145 & 250 & 0.93 & $0.56-1.53$ & 0.77 \\
\hline & $\mathrm{CC}$ & Yes & 35 & 55 & 1.02 & $0.55-1.90$ & 0.95 \\
\hline & $\mathrm{CC}$ & No & 30 & 48 & 1.00 & & \\
\hline \multirow[t]{6}{*}{ rs6804441 } & GG & Yes & 165 & 482 & 0.28 & $0.15-0.54$ & $<0.01$ \\
\hline & GG & No & 180 & 427 & 0.34 & $0.18-0.66$ & $<0.01$ \\
\hline & AG & Yes & 116 & 197 & 0.48 & $0.25-0.94$ & 0.03 \\
\hline & AG & No & 126 & 175 & 0.59 & $0.3-1.14$ & 0.12 \\
\hline & AA & Yes & 21 & 21 & 0.82 & $0.34-1.95$ & 0.65 \\
\hline & AA & No & 22 & 18 & 1.00 & & \\
\hline \multirow[t]{6}{*}{ rs41271391 } & TT & Yes & 88 & 287 & 0.61 & $0.39-0.96$ & 0.03 \\
\hline & TT & No & 117 & 254 & 0.92 & $0.59-1.43$ & 0.71 \\
\hline & GT & Yes & 138 & 322 & 0.86 & $0.56-1.32$ & 0.48 \\
\hline & GT & No & 171 & 286 & 1.20 & $0.78-1.83$ & 0.41 \\
\hline & GG & Yes & 76 & 91 & 1.67 & $1.03-2.72$ & 0.04 \\
\hline & GG & No & 40 & 80 & 1.00 & & \\
\hline
\end{tabular}

0.05 ;OR $=3.78,95 \%$ CI: $1.88-$ 7.61, $P<0.05$ )(Table 8). On the contrary, the rs6804441 GG was associated with depressed RSA risk than AA $(\mathrm{OR}=0.28$, 95\% CI: 0.15-0.54, $P<0.05$; OR $=0.34,95 \%$ CI: 0.18-0.66, $P<0.05)$. Moreover, although night shift contributed to increased incidence of RSA (OR $=1.67,95 \%$ CI: 1.03-2.72, $P<0.05)$, the rs41271391 TT could reverse the single effect of night shift $(\mathrm{OR}=0.61,95 \%$ CI: 0.39-0.96, $P<0.05$ ).

Among the population who frequently stayed up late, carriers of rs3116496 CC (OR $=7.31,95 \%$ CI: 3.32-16.10, $P<$ $0.05)$ possessed a larger possibility of developing RSA than carriers of TC (OR $=2.54,95 \% \mathrm{CI}$ : 1.80-3.59, $P<0.05)$ and TT $(\mathrm{OR}=1.66,95 \% \mathrm{CI}: 1.29-2.15, P<0.05)$ (Table 9). The TC carriers 
Table 9. Interactive effects of CD28/B7 pathway-related SNPs and staying up late on susceptibility to recurrent spontaneous abortion

\begin{tabular}{|c|c|c|c|c|c|c|c|}
\hline SNP & Genotype & Staying up late & Case & Control & OR & $95 \% \mathrm{CI}$ & P value \\
\hline \multirow[t]{6}{*}{ rs3116496 } & CC & Yes & 22 & 9 & 7.31 & $3.32-16.10$ & $<0.01$ \\
\hline & $\mathrm{CC}$ & No & 13 & 21 & 1.85 & $0.91-3.76$ & 0.08 \\
\hline & TC & Yes & 73 & 86 & 2.54 & $1.80-3.59$ & $<0.01$ \\
\hline & TC & No & 148 & 250 & 1.77 & $1.38-2.28$ & $<0.01$ \\
\hline & TT & Yes & 138 & 248 & 1.66 & $1.29-2.15$ & $<0.01$ \\
\hline & TT & No & 236 & 706 & 1.00 & & \\
\hline \multirow[t]{6}{*}{ rs3181098 } & AA & Yes & 61 & 43 & 3.73 & $2.40-5.77$ & $<0.01$ \\
\hline & AA & No & 50 & 148 & 0.89 & $0.61-1.29$ & 0.53 \\
\hline & GA & Yes & 94 & 145 & 1.70 & $1.23-2.36$ & $<0.01$ \\
\hline & GA & No & 213 & 477 & 1.17 & $0.91-1.52$ & 0.22 \\
\hline & GG & Yes & 78 & 155 & 1.32 & $0.94-1.85$ & 0.10 \\
\hline & GG & No & 134 & 352 & 1.00 & & \\
\hline \multirow[t]{6}{*}{ rs3181100 } & CC & Yes & 131 & 157 & 2.95 & $1.80-4.84$ & $<0.01$ \\
\hline & CC & No & 224 & 471 & 1.68 & $1.06-2.68$ & 0.03 \\
\hline & GC & Yes & 89 & 151 & 2.09 & $1.25-3.47$ & $<0.01$ \\
\hline & GC & No & 147 & 414 & 1.26 & $0.78-2.02$ & 0.34 \\
\hline & GG & Yes & 13 & 35 & 1.31 & $0.61-2.84$ & 0.49 \\
\hline & GG & No & 26 & 92 & 1.00 & & \\
\hline \multirow[t]{6}{*}{ rs1915087 } & TT & Yes & 62 & 190 & 0.94 & $0.55-1.61$ & 0.82 \\
\hline & TT & No & 229 & 526 & 1.25 & $0.78-2.03$ & 0.36 \\
\hline & CT & Yes & 131 & 153 & 2.47 & $1.48-4.11$ & $<0.01$ \\
\hline & СT & No & 143 & 379 & 1.09 & $0.66-1.78$ & 0.74 \\
\hline & $\mathrm{CC}$ & Yes & 40 & 31 & 3.72 & $1.93-7.14$ & $<0.01$ \\
\hline & CC & No & 25 & 72 & 1.00 & & \\
\hline \multirow[t]{6}{*}{ rs6804441 } & GG & Yes & 105 & 244 & 0.63 & $0.30-1.32$ & 0.22 \\
\hline & GG & No & 240 & 665 & 0.53 & $0.26-1.08$ & 0.08 \\
\hline & $\mathrm{AG}$ & Yes & 98 & 79 & 1.81 & $0.84-3.90$ & 0.12 \\
\hline & $\mathrm{AG}$ & No & 144 & 293 & 0.72 & $0.35-1.50$ & 0.37 \\
\hline & AA & Yes & 30 & 20 & 2.19 & $0.89-5.42$ & 0.09 \\
\hline & AA & No & 13 & 19 & 1.00 & & \\
\hline \multirow[t]{6}{*}{ rs41271391 } & TT & Yes & 50 & 106 & 0.87 & $0.53-1.45$ & 0.60 \\
\hline & TT & No & 155 & 435 & 0.66 & $0.43-1.01$ & 0.05 \\
\hline & GT & Yes & 108 & 142 & 1.41 & $0.89-2.22$ & 0.14 \\
\hline & GT & No & 201 & 466 & 0.80 & $0.53-1.21$ & 0.29 \\
\hline & GG & Yes & 75 & 95 & 1.46 & $0.90-2.38$ & 0.12 \\
\hline & GG & No & 41 & 76 & 1.00 & & \\
\hline
\end{tabular}

who stayed up early were also liable to suffer from RSA (OR $=1.77,95 \%$ CI: $1.38-2.28, P<$ 0.05). Analogously, genotypes AA and AG of rs3181098 also conferred high incidence of RSA among the population who stayed up late $(\mathrm{OR}=3.73,95 \% \mathrm{CI}: 2.40-5.77, P<0.05 ; \mathrm{OR}=1.70$, $95 \%$ CI: $1.23-2.36, P<0.05)$. The genotypes CC and GC of rs3181100 were also associated with increased risk of RSA among the subjects who stayed up late (OR $=2.95,95 \%$ CI: 1.80 $4.84, P<0.05$; OR $=2.09,95 \% \mathrm{CI}: 1.25-3.47, P<0.05$ ). With the stay-up-late population as the research target, it could be found that genotypes CC and TC of rs 1915087 were, respectively, correlated with incremental susceptibility to RSA (OR $=3.72$, 95\% CI: 1.93-7.14, $P<0.05$; OR $=2.47,95 \%$ CI: $1.48-4.11, P<0.05$ ).

Furthermore, physical exercise was found to positively affect the pregnancy of subjects (OR $=0.58,95 \%$ CI: $0.46-0.74, P<0.05)$, whereas genotypes CC and TC of rs3116496, respectively, led the positive direction to the negative one $\mathrm{OR}=2.89,95 \% \mathrm{CI}: 1.32-6.30$, $P<0.05$; OR $=1.68,95 \%$ CI: $1.23-2.31, P<0.05$ )(Table 10). Carriers of rs3181098 GG were less prone to suffer from RSA when they regularly did physical exercises (OR $=0.68,95 \%$ CI: $0.49-0.95, P<0.05)$. Moreover, the rs6804441 GG carriers were associated with less incidences of RSA whether they frequently experienced physical exercise or not $(\mathrm{OR}=0.20$, 95\% CI: $0.10-0.40, P<0.05 ;$ OR $=0.35,95 \%$ CI: $0.17-0.70, P<0.05$ ). The rs41271391 TT carriers also followed the same trend (OR $=0.33,95 \% \mathrm{CI}$ : $0.22-0.50, P<0.05$; OR $=0.56,95 \%$ CI: $0.37-0.87, P<0.05$ ).

Interestingly, only if the subjects carried the rs3116496 C allele or had a history of miscarriage, they were usually correlated with higher risk of RSA (all $P<0.05$ )(Table 11). Slightly different from rs3116496, people with the miscarriage history had a high possibility to suffer from RSA regardless of their rs3181098 genotypes (all $P<0.05$ ). Besides, miscarriage also conferred ones carrying rs3181100 CC and rs1915087 CT to contract RSA more easily $(\mathrm{OR}=6.53,95 \% \mathrm{CI}: 4.10-10.40, P<0.05 ; \mathrm{OR}=2.56,95 \% \mathrm{CI}: 1.55-4.21, P<0.05)$. The women without experiencing miscarriages were less liable to contract RSA when their genotypes were rs6804441 GG and rs41271391 TT (all $P<0.05$ ). The influences brought about by the 
Table 10. Interactive effects of CD28/B7 pathway-related SNPs and physical exercise on susceptibility to recurrent spontaneous abortion

\begin{tabular}{|c|c|c|c|c|c|c|c|}
\hline SNP & Genotype & Physical exercise & Case & Control & OR & $95 \% \mathrm{CI}$ & P value \\
\hline \multirow{6}{*}{ rs3116496 } & $\mathrm{CC}$ & Yes & 18 & 19 & 1.77 & $0.91-3.46$ & 0.09 \\
\hline & CC & No & 17 & 11 & 2.89 & $1.32-6.30$ & 0.01 \\
\hline & $\mathrm{TC}$ & Yes & 113 & 216 & 0.98 & $0.73-1.31$ & 0.88 \\
\hline & $\mathrm{TC}$ & No & 108 & 120 & 1.68 & $1.23-2.31$ & $<0.01$ \\
\hline & TT & Yes & 192 & 614 & 0.58 & $0.46-0.74$ & $<0.01$ \\
\hline & TT & No & 182 & 340 & 1.00 & & \\
\hline \multirow[t]{6}{*}{ rs3181098 } & AA & Yes & 50 & 113 & 0.84 & $0.56-1.28$ & 0.42 \\
\hline & AA & No & 61 & 78 & 1.49 & $0.98-2.26$ & 0.06 \\
\hline & GA & Yes & 160 & 410 & 0.74 & $0.55-1.01$ & 0.06 \\
\hline & GA & No & 147 & 212 & 1.32 & $0.95-1.83$ & 0.09 \\
\hline & GG & Yes & 117 & 326 & 0.68 & $0.49-0.95$ & 0.02 \\
\hline & GG & No & 95 & 181 & 1.00 & & \\
\hline \multirow[t]{6}{*}{ rs3181100 } & $\mathrm{CC}$ & Yes & 189 & 404 & 1.11 & $0.63-1.95$ & 0.72 \\
\hline & CC & No & 166 & 224 & 1.76 & $0.99-3.11$ & 0.05 \\
\hline & GC & Yes & 114 & 363 & 0.74 & $0.42-1.32$ & 0.31 \\
\hline & GC & No & 122 & 202 & 1.43 & $0.80-2.56$ & 0.23 \\
\hline & GG & Yes & 20 & 82 & 0.58 & $0.28-1.19$ & 0.14 \\
\hline & GG & No & 19 & 45 & 1.00 & & \\
\hline \multirow[t]{6}{*}{ rs1915087 } & $\mathrm{TT}$ & Yes & 150 & 456 & 0.64 & $0.40-1.01$ & 0.06 \\
\hline & $\mathrm{TT}$ & No & 141 & 229 & 1.19 & $0.74-1.92$ & 0.47 \\
\hline & $\mathrm{CT}$ & Yes & 145 & 352 & 0.80 & $0.50-1.27$ & 0.34 \\
\hline & $\mathrm{CT}$ & No & 128 & 180 & 1.38 & $0.85-2.23$ & 0.19 \\
\hline & $\mathrm{CC}$ & Yes & 33 & 41 & 1.56 & $0.83-2.92$ & 0.16 \\
\hline & $\mathrm{CC}$ & No & 32 & 62 & 1.00 & & \\
\hline \multirow[t]{6}{*}{ rs6804441 } & GG & Yes & 177 & 585 & 0.20 & $0.10-0.40$ & $<0.01$ \\
\hline & GG & No & 168 & 324 & 0.35 & $0.17-0.70$ & $<0.01$ \\
\hline & AG & Yes & 124 & 239 & 0.35 & $0.17-0.70$ & $<0.01$ \\
\hline & AG & No & 118 & 133 & 0.59 & $0.29-1.22$ & 0.15 \\
\hline & AA & Yes & 22 & 25 & 0.59 & $0.24-1.42$ & 0.24 \\
\hline & AA & No & 21 & 14 & 1.00 & & \\
\hline \multirow[t]{6}{*}{ rs41271391 } & $\mathrm{TT}$ & Yes & 105 & 348 & 0.33 & $0.22-0.50$ & $<0.01$ \\
\hline & TT & No & 100 & 193 & 0.56 & $0.37-0.87$ & 0.01 \\
\hline & GT & Yes & 158 & 391 & 0.44 & $0.29-0.66$ & $<0.01$ \\
\hline & GT & No & 151 & 217 & 0.76 & $0.50-1.15$ & 0.19 \\
\hline & GG & Yes & 59 & 110 & 0.58 & $0.36-0.95$ & 0.03 \\
\hline & GG & No & 56 & 61 & 1.00 & & \\
\hline
\end{tabular}

combined role of rs3116496/rs3181098 and induced abortions were quite similar to those by rs3116496/rs3181098 and history of miscarriage (Table 12). It could also be observed that history of induced abortion facilitated carriers of rs3181100 CC/GC and rs41271391 GG/TG more readily to suffer from RSA (all $P<0.05$ ).

\section{Discussion}

According to the current study, diverse environmental factors might influence the susceptibility to RSA, such as night shift, history of repeated abortions, history of smoking and intake of vitamin supplement (Table 2-3). In fact, disorders of circadian rhythm and sleep deprivation have been demonstrated to cause a series of diseases, including somnipathy, gastrointestinal neurosis, dyssecretosis of insulin, glucocorticoid, thyroid hormones and sex hormones, hypertension and cardiopathy $[11,12]$. The long-term night shift was also documented to largely elevate the possibility of suffering from diverse cancers (e.g. breast cancer, ovarian cancer, gastric cancer and intestinal cancer) and RSA [13, 14]. In addition, the repeated abortions often easily induced such gynecological diseases as endometrial base damage, endometritis and intrauterine adhesion, and the generated excessive mental pressure also increased subjects' risk of subsequent pregnancies [15-18]. Furthermore, the toxic substances inside cigarettes could seriously impact the quality of eggs, as well as hinder the division, implantation and growth of embryonic cells, finally leading to loss or dysplasia of embryos. Therefore, either passive smoking or active smoking was the risk parameter of abnormal pregnancies [19]. Moreover, the females all increasingly demanded essential micro-elements, yet $70 \%$ of the pregnant women had severe nausea and vomiting of pregnancy, which all accelerated the loss of the micro-elements. And it has been documented that low levels of zinc, copper, iron and selenium could cease the embryonic development and lead to abortion [20]. Furthermore, VD maintained the regular functioning of humoral immunity not merely through keeping the stability of CD4/CD8 ratio, but also by holding the TH1/TH2 immunity in a normal range $[21,22]$. Based on the mechanisms, Harvey et al. 


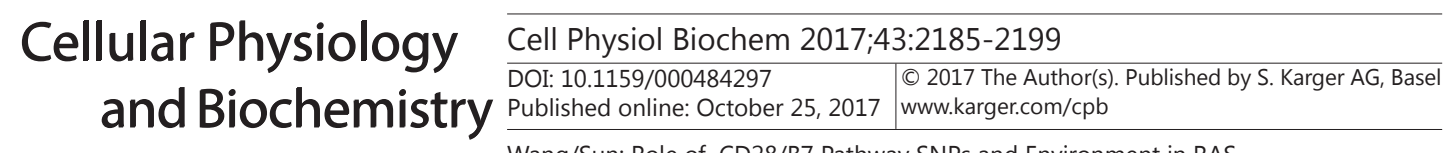
2195

Wang/Sun: Role of CD28/B7 Pathway SNPs and Environment in RAS

Table 11. Interactive effects of CD28/B7 pathway-related SNPs and history of miscarriage on susceptibility to recurrent spontaneous abortion

\begin{tabular}{|c|c|c|c|c|c|c|c|}
\hline SNP & Genotype & Miscarriage & Case & Control & OR & $95 \%$ CI & $P$ value \\
\hline \multirow[t]{6}{*}{ rs3116496 } & $\mathrm{CC}$ & Yes & 25 & 16 & 6.02 & $3.15-11.50$ & $<0.01$ \\
\hline & CC & No & 10 & 14 & 2.75 & $1.20-6.29$ & 0.01 \\
\hline & $\mathrm{TC}$ & Yes & 133 & 137 & 3.74 & $2.80-4.98$ & $<0.01$ \\
\hline & TC & No & 88 & 199 & 1.70 & $1.26-2.29$ & $<0.01$ \\
\hline & TT & Yes & 186 & 230 & 3.11 & $2.42-4.00$ & $<0.01$ \\
\hline & $\mathrm{TT}$ & No & 188 & 724 & 1.00 & & \\
\hline \multirow[t]{6}{*}{ rs3181098 } & AA & Yes & 67 & 39 & 4.72 & $3.02-7.38$ & $<0.01$ \\
\hline & AA & No & 42 & 152 & 0.76 & $0.51-1.13$ & 0.18 \\
\hline & GA & Yes & 183 & 161 & 3.12 & $2.31-4.21$ & $<0.01$ \\
\hline & GA & No & 126 & 461 & 0.75 & $0.56-1.00$ & 0.05 \\
\hline & GG & Yes & 94 & 183 & 1.41 & $1.02-1.95$ & 0.04 \\
\hline & GG & No & 118 & 324 & 1.00 & & \\
\hline \multirow[t]{6}{*}{ rs3181100 } & $\mathrm{CC}$ & Yes & 223 & 115 & 6.53 & $4.10-10.40$ & $<0.01$ \\
\hline & CC & No & 132 & 513 & 0.87 & $0.55-1.36$ & 0.53 \\
\hline & GC & Yes & 112 & 242 & 1.56 & $0.98-2.48$ & 0.06 \\
\hline & GC & No & 124 & 323 & 1.29 & $0.82-2.04$ & 0.27 \\
\hline & GG & Yes & 9 & 26 & 1.17 & $0.49-2.76$ & 0.73 \\
\hline & GG & No & 30 & 101 & 1.00 & & \\
\hline \multirow[t]{6}{*}{ rs1915087 } & TT & Yes & 128 & 190 & 1.44 & $0.87-2.38$ & 0.15 \\
\hline & TT & No & 143 & 491 & 0.62 & $0.38-1.01$ & 0.06 \\
\hline & CT & Yes & 179 & 150 & 2.56 & $1.55-4.21$ & $<0.01$ \\
\hline & CT & No & 115 & 386 & 0.64 & $0.39-1.05$ & 0.07 \\
\hline & $\mathrm{CC}$ & Yes & 37 & 43 & 1.84 & $0.98-3.46$ & 0.06 \\
\hline & CC & No & 28 & 60 & 1.00 & & \\
\hline \multirow[t]{6}{*}{ rs6804441 } & GG & Yes & 187 & 270 & 0.92 & $0.49-1.75$ & 0.81 \\
\hline & GG & No & 158 & 639 & 0.33 & $0.17-0.62$ & $<0.01$ \\
\hline & AG & Yes & 132 & 98 & 1.80 & $0.92-3.49$ & 0.08 \\
\hline & AG & No & 110 & 274 & 0.54 & $0.28-1.03$ & 0.06 \\
\hline & AA & Yes & 25 & 15 & 2.22 & $0.92-5.38$ & 0.08 \\
\hline & AA & No & 18 & 24 & 1.00 & & \\
\hline \multirow[t]{6}{*}{ rs41271391 } & TT & Yes & 111 & 142 & 1.39 & $0.91-2.13$ & 0.12 \\
\hline & TT & No & 94 & 399 & 0.42 & $0.28-0.63$ & $<0.01$ \\
\hline & GT & Yes & 168 & 161 & 1.86 & $1.24-2.79$ & $<0.01$ \\
\hline & GT & No & 141 & 447 & 0.56 & $0.38-0.83$ & $<0.01$ \\
\hline & GG & Yes & 65 & 80 & 1.45 & $0.90-2.33$ & 0.12 \\
\hline & GG & No & 51 & 91 & 1.00 & & \\
\hline
\end{tabular}

Table 12. Interactive effects of CD28/B7 pathway-related SNPs and history of induced abortion on susceptibility to recurrent spontaneous abortion

\begin{tabular}{|c|c|c|c|c|c|c|c|}
\hline SNP & Genotype & Induced abortion & Case & Control & OR & $95 \%$ CI & $\mathrm{P}$ value \\
\hline \multirow[t]{6}{*}{ rs3116496 } & $\mathrm{CC}$ & Yes & 16 & 23 & 2.33 & $1.21-4.49$ & 0.01 \\
\hline & $\mathrm{CC}$ & No & 19 & 7 & 9.08 & $3.77-21.91$ & $<0.01$ \\
\hline & $\mathrm{TC}$ & Yes & 124 & 126 & 3.29 & $2.46-4.41$ & $<0.01$ \\
\hline & $\mathrm{TC}$ & No & 97 & 210 & 1.55 & $1.16-2.06$ & $<0.01$ \\
\hline & TT & Yes & 169 & 268 & 2.11 & $1.65-2.70$ & $<0.01$ \\
\hline & TT & No & 205 & 686 & 1.00 & & \\
\hline \multirow[t]{6}{*}{ rs3181098 } & AA & Yes & 61 & 60 & 2.80 & $1.86-4.22$ & $<0.01$ \\
\hline & AA & No & 50 & 131 & 1.05 & $0.72-1.54$ & 0.80 \\
\hline & GA & Yes & 162 & 197 & 2.26 & $1.69-3.03$ & $<0.01$ \\
\hline & GA & No & 145 & 425 & 0.94 & $0.71-1.24$ & 0.66 \\
\hline & GG & Yes & 86 & 160 & 1.48 & $1.06-2.06$ & 0.02 \\
\hline & GG & No & 126 & 347 & 1.00 & & \\
\hline \multirow[t]{6}{*}{ rs3181100 } & $\mathrm{CC}$ & Yes & 160 & 203 & 2.50 & $1.58-3.95$ & $<0.01$ \\
\hline & $\mathrm{CC}$ & No & 195 & 425 & 1.45 & $0.93-2.27$ & 0.10 \\
\hline & GC & Yes & 140 & 182 & 2.44 & $1.53-3.88$ & $<0.01$ \\
\hline & GC & No & 96 & 383 & 0.79 & $0.50-1.27$ & 0.33 \\
\hline & GG & Yes & 9 & 32 & 0.89 & $0.38-2.08$ & 0.79 \\
\hline & GG & No & 30 & 95 & 1.00 & & \\
\hline \multirow[t]{6}{*}{ rs1915087 } & TT & Yes & 142 & 209 & 1.38 & $0.87-2.19$ & 0.17 \\
\hline & TT & No & 149 & 476 & 0.64 & $0.41-1.00$ & 0.05 \\
\hline & $\mathrm{CT}$ & Yes & 136 & 174 & 1.59 & $0.99-2.53$ & 0.05 \\
\hline & CT & No & 138 & 358 & 0.78 & $0.50-1.23$ & 0.29 \\
\hline & $\mathrm{CC}$ & Yes & 31 & 34 & 1.85 & $0.98-3.50$ & 0.06 \\
\hline & $\mathrm{CC}$ & No & 34 & 69 & 1.00 & & \\
\hline \multirow[t]{6}{*}{ rs6804441 } & GG & Yes & 145 & 287 & 0.74 & $0.37-1.47$ & 0.39 \\
\hline & GG & No & 215 & 622 & 0.51 & $0.26-1.00$ & 0.05 \\
\hline & AG & Yes & 136 & 113 & 1.77 & $0.87-3.56$ & 0.11 \\
\hline & $A G$ & No & 91 & 259 & 0.52 & $0.26-1.04$ & 0.06 \\
\hline & AA & Yes & 28 & 17 & 2.42 & $0.99-5.89$ & 0.05 \\
\hline & AA & No & 15 & 22 & 1.00 & & \\
\hline \multirow[t]{6}{*}{ rs41271391 } & TT & Yes & 87 & 140 & 1.28 & $0.84-1.96$ & 0.26 \\
\hline & TT & No & 118 & 401 & 0.61 & $0.41-0.89$ & 0.01 \\
\hline & GT & Yes & 158 & 213 & 1.53 & $1.03-2.25$ & 0.03 \\
\hline & GT & No & 151 & 395 & 0.79 & $0.54-1.15$ & 0.22 \\
\hline & GG & Yes & 64 & 64 & 2.06 & $1.27-3.32$ & $<0.01$ \\
\hline & GG & No & 52 & 107 & 1.00 & & \\
\hline
\end{tabular}

proposed that VD deficiency during pregnancy could cause permanently changed forms and functions of offsprings' immune organs [23, 24]. Finally, the in-vitro addition of fruits and 


\section{Cellular Physiology Cell Physiol Biochem 2017;43:2185-2199 \begin{tabular}{l|l|l} 
DOI: 10.1159/000484297 & (0) 2017 The Author(s). Published by S. Karger AG, Basel \\
www.karger.com/cpb
\end{tabular}}

Wang/Sun: Role of CD28/B7 Pathway SNPs and Environment in RAS

vegetables were demonstrated to positively stimulate IL-2, IFN-nstrated to $\beta$ via modulating the secretion of Th1/Th2 cytokines $[25,26]$. The underlying mechanism could be roughly summarized as that fruits and vegetables were rich in vitamin A, vitamin $\mathrm{C}$, vitamin $\mathrm{E}$ and micro-elements, the lacking of which might contribute to the damaged immune system of organisms [27-30]. For instance, enough supply of vitamin A could promptly raise the number of peripheral blood lymphocytes, and vitamin E exerted an inhibitory role in the production of prostaglandin E2 (PGE2), the substance that suppressed the organisms' immune functions in a non-specific manner [31-33]. All in all, due to the linkage between immunity dysfunction and elevated prevalence of RSA, proper intake of fruit and vegetable were beneficial living habits against abortion.

In addition, it was previously documented that B7-1 (i.e. CD80) and B7-2 (e.g. CD86) were mainly expressed in the activated dendritic cells and macrophages, and they were the natural ligands of CD28. The combination of CD28 and B7 would boost the expressions of interleukin-2 (IL-2), IL-4 and IL-5, and this auxo-action would be enhanced when the second messenger existed [34]. Besides, the co-stimulatory signals of CD28 were involved in multiple intracellular pathways that modified the immunity responses, including PI3K pathway, GRB-2/SOS-related p21Ras pathway and ITK/EMT pathway [35-37]. Since one contributor of RSA was regarded as the immunity dysfunction, it was taken for granted that the mal adjusted CD28/B7 pathway was the vital reason for RSA development. Consistent with the hypothesis, hindering B7-1 and B7-2 expressions could hold up the maternal rejection to allogenic fetus within matings that were prone to suffer from miscarriages [38]. Moreover, methods have been explored to avoid the abortion relevant to dysfunctional immunity via altering the costimulatory signal mediated by CD28 [39]. Accordingly, the immunity-related SNPs situated within CD28 and B7 were also estimated as the risk factor for RSA. To be specific, rs1129055 of B7-2 was remarkably associated with the altered risk of pneumonia-induced sepsis [40]. B7-4 rs6804441 was also investigated whether it was correlated with systemic lupus erythematosus among an Asian population [41]. A prospective study based on the Polish population also revealed the relationship between CD28 rs3116496/ rs3181098 and cancer risk [42]. Above all, the SNPs that showed statistical significances could be deemed as the biomarkers for RSA development (Table 3-4). Furthermore, the interactions of SNPs and environment also derived the results that differed from SNPs and environment alone (Table 5-11)[43, 44].

Nonetheless, this study was limited by several factors. Firstly, recall bias due to memory distortion or incomplete memory would give rise to the systematic error existing between memory and the truth [45]. Secondly, the distinct outpatient rate caused by the different subjects' knowledge of the diseases would magnify the selection bias when patients were selected from the hospital. Thirdly, the sample size included was not enough for generalizing the study results into other populations. Moreover, only finite factors were investigated in this study, other environmental factors, such as coffee, were not investigated. What's more, some women might conceal their reproductive history because of the one-child policy in China, so the breeding conditions might not be a bit distinct from the real situation. Finally, we only studied the superficial association of environmental and genetic factors with risk of RSA, but we did not explore the potential molecular mechanisms underlying RSA. Hence, more in-depth studies with a large scale were demanded for the following researches.

\section{Conclusion}

The environment factors, including night shift, history of repeated abortions, history of smoking and intake of vitamin supplement, could interact with SNPs located within CD28/ B7 pathway (e.g. rs3116496, rs6804441 and rs1129055) to modify the risk of RSA among the Chinese population. 


\section{Cellular Physiology Cell Physiol Biochem 2017;43:2185-2199 \begin{tabular}{ll|l} 
DOI: 10.1159/000484297 & O 2017 The Author(s). Published by S. Karger AG, Basel \\
www.karger.com/cpb
\end{tabular} \\ Wang/Sun: Role of CD28/B7 Pathway SNPs and Environment in RAS}

\section{Funding}

This work was supported in part by grants from Shandong medical and health science and technology development program (ID: 2013WS0298).

\section{Acknowledgements}

None.

\section{Disclosure Statement}

The authors declare that they have no competing interests.

\section{References}

1 Daya S, Gunby J, Clark DA: Intravenous immunoglobulin therapy for recurrent spontaneous abortion: a meta-analysis. Am J Reprod Immunol 1998;39:69-76.

-2 Zhao H, Miao Z, Wei X: Progress of Diagnosis and Treatment of Recurrent Miscarriage. Clin Med 2013;33:104-108.

-3 Regan L, Braude PR, Trembath PL: Influence of past reproductive performance on risk of spontaneous abortion. BMJ 1989;299:541-545.

4 Sanguansermsri D, Pongcharoen S: Pregnancy immunology: decidual immune cells. Asian Pac J Allergy Immunol 2008;26:171-181.

5 Zhang S, Audard V, Fan Q Pawlak A, Lang P, Sahali D: Immunopathogenesis of idiopathic nephrotic syndrome. Contrib Nephrol 2011;169:94-106.

6 Gan L, Jia R, Zhou L, Guo J, Fan M: Fusion of CTLA-4 with HPV16 E7 and E6 enhanced the potency of therapeutic HPV DNA vaccine. PLoS One 2014;9:e108892.

7 Li X, Dai J, Yuan H, Zhang X: Expression and role of CD40/CD40L and B7-1/CD28 interaction in folic acidinduced nephropathy. Chinese J Nephrol 2005;21:534-537.

-8 Eblen AC, Gercel-Taylor C, Shields LB, Sanfilippo JS, Nakajima ST, Taylor DD: Alterations in humoral immune responses associated with recurrent pregnancy loss. Fertil Steril 2000;73:305-313.

-9 Latini G, De Felice C, Presta G, Del Vecchio A, Paris I, Ruggieri F, Mazzeo P: In utero exposure to di-(2ethylhexyl)phthalate and duration of human pregnancy. Environ Health Perspect 2003;111:1783-1785.

10 Lin X, Wang Z, Wang M, Wu D: Changes of Menstrual Function of Female Workers in Plastic Plants. J Envir Health 2003;20:98-100.

-11 Jia Y, Lu Y, Wu K, Lin Q Shen W, Zhu M, Huang S, Chen J: Does night work increase the risk of breast cancer? A systematic review and meta-analysis of epidemiological studies. Cancer Epidemiol 2013;37:197-206.

12 Poole EM, Schernhammer ES, Tworoger SS: Rotating night shift work and risk of ovarian cancer. Cancer Epidemiol Biomark Prev 2011;20:934-938.

13 Whelan EA, Lawson CC, Grajewski B, Hibert EN, Spiegelman D, Rich-Edwards JW: Work schedule during pregnancy and spontaneous abortion. Epidemiology 2007;18:350-355.

14 Irwin M, McClintick J, Costlow C, Fortner M, White J, Gillin JC: Partial night sleep deprivation reduces natural killer and cellular immune responses in humans. FASEB J 1996;10:643-653.

15 Sun Y, Che Y, Gao E, Olsen J, Zhou W: Induced abortion and risk of subsequent miscarriage. Int J Epidemiol 2003;32:449-454.

16 Lv F, Xu X, Zhang S, Wang L, Wang N, He B, Wang J: Repeated abortion affects subsequent pregnancy outcomes in BALB/c mice. PLoS One 2012;7:e48384.

17 Virk J, Zhang J, Olsen J: Medical abortion and the risk of subsequent adverse pregnancy outcomes. N Engl J Med 2007;357:648-653. 


\section{Cellular Physiology Cell Physiol Biochem 2017;43:2185-2199

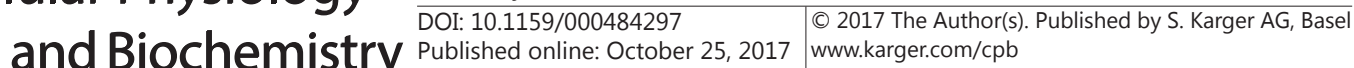

18 Maconochie N, Doyle P, Prior S, Simmons R: Risk factors for first trimester miscarriage--results from a UKpopulation-based case-control study. BJOG 2007;114:170-186.

19 Harlap S, Shiono PH: Alcohol, smoking, and incidence of spontaneous abortions in the first and second trimester. Lancet 1980;2:173-176.

20 Wilson RD, Johnson JA, Wyatt P, Allen V, Gagnon A, Langlois S, Blight C, Audibert F, Desilets V, Brock JA, Koren G, Goh YI, Nguyen P, Kapur B, Genetics Committee of the Society of O, Gynaecologists of C, The Motherrisk P: Pre-conceptional vitamin/folic acid supplementation 2007: the use of folic acid in combination with a multivitamin supplement for the prevention of neural tube defects and other congenital anomalies. J Obstet Gynaecol Can 2007;29:1003-1026.

-21 Cantorna MT, Zhu Y, Froicu M, Wittke A: Vitamin D status, 1, 25-dihydroxyvitamin D3, and the immune system. Am J Clin Nutr 2004;80:1717S-1720S.

22 Cantorna MT, Mahon BD: Mounting evidence for vitamin D as an environmental factor affecting autoimmune disease prevalence. Exp Biol Med (Maywood) 2004;229:1136-1142.

23 Harvey L, Burne TH, McGrath JJ, Eyles DW: Developmental vitamin D3 deficiency induces alterations in immune organ morphology and function in adult offspring. J Steroid Biochem Mol Biol 2010;121:239-242.

24 Chen A, Li C, Wang J, Sha H, Piao S, Liu S: Role of Toll-Like Receptor 3 Gene Polymorphisms in Preeclampsia. Cell Physiol Biochem 2015;37:1927-1933.

25 Lin JY, Tang CY: Total phenolic contents in selected fruit and vegetable juices exhibit a positive correlation with interferon- $\gamma$, interleukin-5, and interleukin-2 secretions using primary mouse splenocytes. J Food Comp Anal 2008;21:45-53.

26 Ogawa K, Nakada K, Yamashita S, Hasegawa T, Moriguchi S: Beneficial effects of the vegetable juice Aojiru on cellular immunity in Japanese young women. Nutrit Res 2004;24:613-620.

-27 Folchetti LD, Monfort-Pires M, de Barros CR, Martini LA, Ferreira SR: Association of fruits and vegetables consumption and related-vitamins with inflammatory and oxidative stress markers in prediabetic individuals. Diabetol Metab Syndr 2014;6:22.

28 Mirfendereski E, Jahanian R: Effects of dietary organic chromium and vitamin C supplementation on performance, immune responses, blood metabolites, and stress status of laying hens subjected to high stocking density. Poult Sci 2015;94:281-288.

29 Pan JH, Feng L, Jiang WD, Wu P, Kuang SY, Tang L, Zhang YA, Zhou XQ Liu Y: Vitamin E deficiency depressed fish growth, disease resistance, and the immunity and structural integrity of immune organs in grass carp (Ctenopharyngodon idella): Referring to NF-kappaB, TOR and Nrf2 signaling. Fish Shellfish Immunol 2017;60:219-236.

30 Gao H, Liu C, Lin P, Xu L, Li X, Chen Y, Cheng B, Li A, Liu S: Effects of GSTP1 and GPX1 Polymorphisms on the Risk of Preeclampsia in Chinese Han Women. Cell Physiol Biochem 2016;39:2025-2032.

-31 Uzenbayeva LB, Bashnikova IV, Kizhina AG, Ilyina TN, Ilyukha VA, Tyutyunnik NN: [Morphological peculiarities of peripheral blood lymphocytes in arctic foxes after administration of various doses of vitamins A and E]. Morfologiia 2013;143:39-44.

-32 Yano T, Yano Y, Uchida M, Murakami A, Hagiwara K, Otani S, Ichikawa T: The modulation effect of vitamin $\mathrm{E}$ on prostaglandin E2 level and ornithine decarboxylase activity at the promotion phase of lung tumorigenesis in mice. Biochem Pharmacol 1997;53:1757-1759.

-33 Goverse G, Olivier BJ, Molenaar R, Knippenberg M, Greuter M, Konijn T, Cook EC, Beijer MR, Fedor DM, den Haan JM, Napoli JL, Bouma G, Mebius RE: Vitamin A metabolism and mucosal immune function are distinct between BALB/c and C57BL/6 mice. Eur J Immunol 2015;45:89-100.

-34 McAdam AJ, Schweitzer AN, Sharpe AH: The role of B7 co-stimulation in activation and differentiation of CD4+ and CD8+ T cells. Immunol Rev 1998;165:231-247.

-35 Garcon F, Patton DT, Emery JL, Hirsch E, Rottapel R, Sasaki T, Okkenhaug K: CD28 provides T-cell costimulation and enhances PI3K activity at the immune synapse independently of its capacity to interact with the p85/p110 heterodimer. Blood 2008;111:1464-1471.

-36 Schneider H, Cai YC, Prasad KV, Shoelson SE, Rudd CE: T cell antigen CD28 binds to the GRB-2/SOS complex, regulators of p21ras. Eur J Immunol 1995;25:1044-1050.

37 Marengere LE, Okkenhaug K, Clavreul A, Couez D, Gibson S, Mills GB, Mak TW, Rottapel R: The SH3 domain of Itk/Emt binds to proline-rich sequences in the cytoplasmic domain of the T cell costimulatory receptor CD28. J Immunol 1997;159:3220-3229. 


\section{Cellular Physiology Cell Physiol Biochem 2017;43:2185-2199

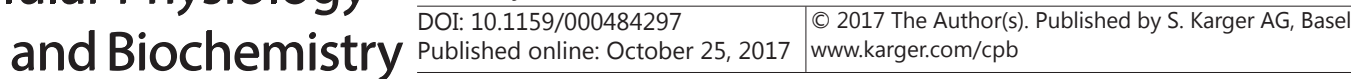 \\ Wang/Sun: Role of CD28/B7 Pathway SNPs and Environment in RAS}

-38 Jin LP, Zhou YH, Wang MY, Zhu XY, Li DJ: Blockade of CD80 and CD86 at the time of implantation inhibits maternal rejection to the allogeneic fetus in abortion-prone matings. J Reprod Immunol 2005;65:133-146.

39 Ling V, Gray G S, Keith J C: Methods of preventing immune-mediated abortion by inhibiting a CD28mediated costimulatory signal. US 2003.

40 Wang C, Gui Q, Zhang K: Functional polymorphisms in CD86 gene are associated with susceptibility to pneumonia-induced sepsis. APMIS 2015;123:433-438.

-41 Zhang Y, Yang J, Zhang J, Sun L, Hirankarn N, Pan HF, Lau CS, Chan TM, Lee TL, Leung AM, Mok CC, Zhang L, Wang Y, Shen JJ, Wong SN, Lee KW, Ho MH, Lee PP, Chung BH, Chong CY, Wong RW, Mok MY, Wong WH, Tong KL, Tse NK, Li XP, Avihingsanon Y, Rianthavorn P, Deekajorndej T, Suphapeetiporn K, Shotelersuk V, Ying SK, Fung SK, Lai WM, Wong CM, Ng IO, Garcia-Barcelo MM, Cherny SS, Cui Y, Sham PC, Yang S, Ye DQ Zhang XJ, Lau YL, Yang W: Genome-wide search followed by replication reveals genetic interaction of CD80 and ALOX5AP associated with systemic lupus erythematosus in Asian populations. Ann Rheum Dis 2016;75:891-898.

42 Tupikowski K, Partyka A, Kolodziej A, Dembowski J, Debinski P, Halon A, Zdrojowy R, Frydecka I, Karabon L: CTLA-4 and CD28 genes' polymorphisms and renal cell carcinoma susceptibility in the Polish population--a prospective study. Tissue Antigens 2015;86:353-361.

43 Manning AK, LaValley M, Liu CT, Rice K, An P, Liu Y, Miljkovic I, Rasmussen-Torvik L, Harris TB, Province MA, Borecki IB, Florez JC, Meigs JB, Cupples LA, Dupuis J: Meta-analysis of gene-environment interaction: joint estimation of SNP and SNP x environment regression coefficients. Genet Epidemiol 2011;35:11-18.

44 Lindstrom S, Schumacher F, Siddiq A, Travis RC, Campa D, Berndt SI, Diver WR, Severi G, Allen N, Andriole G, Bueno-de-Mesquita B, Chanock SJ, Crawford D, Gaziano JM, Giles GG, Giovannucci E, Guo C, Haiman CA, Hayes RB, Halkjaer J, Hunter DJ, Johansson M, Kaaks R, Kolonel LN, Navarro C, Riboli E, Sacerdote C, Stampfer M, Stram DO, Thun MJ, Trichopoulos D, Virtamo J, Weinstein SJ, Yeager M, Henderson B, Ma J, Le Marchand L, Albanes D, Kraft P: Characterizing associations and SNP-environment interactions for GWASidentified prostate cancer risk markers--results from BPC3. PLoS One 2011;6:e17142.

45 Buka SL, Goldstein JM, Seidman LJ, Tsuang MT: Maternal recall of pregnancy history: accuracy and bias in schizophrenia research. Schizophr Bull 2000;26:335-350. 